\title{
La afordancia como herramienta epistémica y ontológica en la demarcación de los sistemas cognitivos desde las posturas situadas ${ }^{*}$
}

\author{
Affordances as Ontologic and Epistemic Tools in \\ the Demarcation of Cognitive Systems from Situated Approaches
}

Melina Gastélum Vargas ${ }^{\dagger}$

\begin{abstract}
Resumen
En la primera parte de este trabajo se da cuenta de ciertas demarcaciones que surgen cuando entendemos la cognición de una manera situada, sobre todo en una postura ecológica. Para ello, pretendo explicar brevemente las implicaciones de hablar de cognición situada, además de comprender la separación entre las visiones extendida y enculturada de la cognición, explicando en términos ontológicos y epistémicos las consecuencias que conlleva el hablar de los sistemas cognitivos en uno u otro sentido. En la segunda parte se propondrá como herramienta epistémica y ontológica a la afordancia para explicar fenómenos que se dan precisamente en estas fronteras difusas entre los sistemas cognitivos y el ambiente, explicando algunas posturas respecto a ella y decantándonos por una postura relacional por su aplicación para fenómenos cognitivos, particularmente la percepción y la cognición social.
\end{abstract}

Palabras clave: afordancia - cognición situada - cognición social - mente extendida - emergencia

\begin{abstract}
In this work I pretend, in the first part, to give an account of certain demarcations that surge when we understand cognition in a situated way, specially in an ecological posture. For that I intend to explain briefly the implications of talking about situated cognition and also understanding the separation between the extended and enculturated postures of cognition; explaining in ontological and epistemic terms the consequences that imply taking one or the other as a theory to explain cognition. In the second part, I will propose affordance as an epistemic and ontological tool to explain phenomena that take place precisely in the diffuse frontiers between the cognitive systems and the environment, explaining some historic ways of understanding the term and favoring a relational posture that allows a good application for understanding cognitive phenomena, in particular perception and social cognition.
\end{abstract}

Keywords: affordance - situated cognition - social cognition - extended mind - emergency

\footnotetext{
* Recibido: 10 de Junio de 2014. Aceptado en versión revisada: 30 de Agosto de 2014.

† Facultad de Filosofía y Letras, UNAM, México. Para contactar a la autora, por favor, escribir a: megava@gmail.com. Metatheoria 5(1)(2014): 145-158. ISSN 1853-2322.

(C) Editorial de la Universidad Nacional de Tres de Febrero. Publicado en la República Argentina.
} 


\section{Introducción}

Dentro de la filosofía de la biología existen diversos temas que son difíciles de categorizar de una manera estricta, sobre todo tomando en cuenta que las fronteras entre muchas disciplinas referentes a lo vivo se han disipado. Dentro de la Biología, podemos destacar que muchos de los estudios que se han hecho acerca del comportamiento de distintos animales generan amplio conocimiento acerca de cómo funciona el aparato cognitivo humano, sobre todo al observar sus interacciones en el ambiente. Cuando hablamos del sistema cognitivo humano existe una pregunta que tiene un lado ontológico y otro epistemológicamente relevante: ¿Dónde acaba ese fenómeno que llamamos mente y dónde empieza el resto del mundo? La pregunta invita a muchas respuestas que se adhieren a debates que tienen que ver con cómo los sistemas cognitivos se relacionan con el ambiente, cómo es que se da el fenómeno de la percepción y sus contenidos, cómo es que interactuamos con otros seres; todo ello tomando en cuenta que la base empírica de dichas preguntas tiene lugar en estudios biológicos, etológicos y antropológicos que permiten al filósofo teorizar en estas demarcaciones. Algunas personas aceptan las demarcaciones de la piel y el cráneo para discernir entre el "nosotros" y el ambiente e incluso afirman que lo que se halla fuera del cuerpo está también fuera de la mente. Otros, sin embargo, se inclinan por el argumento de que los significados de las palabras "simplemente no están en la cabeza" y sostienen un externalismo del significado.

Sin embargo, la mirada que ha surgido en los últimos años y que es la que nos interesa explorar como una corriente muy importante en las ciencias cognitivas, sostiene que los procesos cognitivos están enraizados profundamente en las interacciones que el cuerpo tiene con el entorno y con otros seres vivos. Esta postura guarda dentro de ella muchos supuestos que plantean distinciones en las diversas maneras de aproximarse a ella, algunas más controversiales que otras, sobre todo cuando las cuestiones ontológicas que implican se llevan a sus máximas consecuencias.

En este trabajo pretendo dar cuenta en la primera parte de las demarcaciones que separan una visión de la cognición extendida y una visión enculturada; además de explicar en términos ontológicos y epistémicos las consecuencias que conlleva el hablar de los sistemas cognitivos en uno u otro sentido. La mirada liberal, que es la que se defenderá, considera que la cognición es socialmente extensiva en un modo que va más allá de los ejemplos típicos (como cuadernos y varias tecnologías) y en un modo que propone que la cognición conlleva procesos enactivos dinámicos (como afordancias sociales) en vez de relaciones funcionales. Estas últimas son ontológicamente radicales, pero siguen conservando la idea de la mente de las ciencias cognitivas clásicas pues literalmente conciben que los procesos fuera de la cabeza que cuentan como cognitivos solo son los que podrían ser logrados en la cabeza. Entonces, se queda la noción de proceso cognitivo "en la cabeza", lo cual sigue pareciéndose mucho al modelo estándar, del cual trataremos de deshacernos por las implicaciones que tiene. En la segunda parte se hablará de las afordancias como herramientas epistémicas y ontológicas para entender fenómenos cognitivos justamente en esta relación entre los cuerpos y el ambiente. Básicamente, se explicarán algunas posturas históricas respecto a ella y optaremos por una postura relacional por su aplicación para fenómenos cognitivos, usando en particular los ejemplos de la percepción y la cognición social. Se verá entonces que se puede distinguir entre afordancias ecológicas e interpersonales, tomando en cuenta que la misma percepción ecológica puede requerir habilidades sociales. Creemos que aunque la idea de afordancia gibsoniana se ha discutido mucho, aún falta poner atención al rol que la cognición social juega en la percepción de afordancias ecológicas.

\section{Cognición extendida y enculturada}

La mirada que ha surgido en los últimos años como una corriente muy importante en la filosofía de las ciencias cognitivas sostiene que los procesos cognitivos están enraizados profundamente en las interacciones que el cuerpo tiene con el entorno. Esta postura se contrapone a la llamada postura del Establishment, término que se tomó desde el trabajo de Jerry A. Fodor y Zenon W. Pylyshyn del 2002, que corresponde a entender la cognición sin una real consideración de los sistemas perceptuales y 
motores, que no se consideran relevantes para entender los procesos cognitivos centrales, y más bien son sistemas periféricos que traen información de entrada y de salida a un procesador de información central. Lo que las nuevas corrientes toman como punto de partida teórico no es una mente trabajando o resolviendo problemas abstractos sino un cuerpo que requiere de una mente para que funcione en un ambiente dado. De acuerdo a las nuevas aproximaciones los procesos cognitivos no están limitados a procesamientos simbólicos de estructuras de información internas (a las que solemos llamar representaciones mentales), sino implementadas en varios procesos sensomotores como la motricidad, la percepción, las emociones, la imaginación y la simulación; y varios sustratos físicos (miembros corporales, cuerpos, artefactos, regularidades ambientales).

El tema general de estas aproximaciones es el de la encarnación de la cognición: tanto en el cuerpo como en el mundo. Si bien uno puede distinguir conceptualmente el cerebro del cuerpo y del ambiente, hay un continuo flujo de información que los amalgama juntos. Ya sea que hablemos de resolución de problemas, de percepción o de toma de decisiones, muchos procesos cognitivos conllevan diversidad de procesos que no se hallan solamente en el cerebro. Ahora bien, mientras que la cognición encarnada se refiere a la incorporación del cerebro en el cuerpo y a la integración funcional que todo el complejo cuerpo-cerebro juega en la acción-percepción y en todas las actividades cognitivas de que forma parte. La cognición situada, por su parte, se refiere a la encarnación o incrustación del complejo cuerpo-cerebro en el ambiente (social y ecológico). Se considera que así como nuestros cerebros pueden "consultar" procesos viscerales, también pueden usar estructuras externas o, como Sanjay Chandrasekharan y Terrence C. Stewart (2007) los llaman, estructuras epistémicas. Sin embargo estas estructuras no son sólo apéndices convenientes sino que están inherentemente unidas a nuestra vida mental: forman parte de lo que llamamos "sistema cognitivo". Andy Clark (1999) le llama a estas regularidades ambientales y sociales que soportan y extienden la cognición andamiajes externos (del inglés scaffoldings). Crecemos, aprendemos y actuamos todos los días a través de ellos y además, entre éstos podemos nombrar al lenguaje como el máximo artefacto cognitivo que forma parte de nuestras prácticas cotidianas como seres humanos.

Así, el lenguaje, las culturas y las instituciones permiten a nuestra especie extender los procesos cognitivos más allá del cerebro, del cuerpo y del ambiente inmediato. La cognición entonces no está solo encarnada y situada sino que además está distribuida en el ambiente. En algunos casos incluso colectivos como equipos, familias, grupos y organizaciones procesan información conjuntamente e implementan actividades cognitivas en donde no se puede rastrear una unidad en particular que las implemente como un todo.

La cognición está entonces encarnada, situada en un ambiente y distribuida entre agentes, artefactos y estructuras externas. La dinámica sensomotora constante, el rol de la acción en la percepción y en el aprendizaje, el acoplamiento agente/ambiente y la inteligencia colectiva son determinantes cognitivos que uno debe tomar en cuenta para describir adecuadamente sistemas cognitivos naturales y para construir sistemas cognitivos artificiales.

\subsection{Distinciones}

En la cognición corporizada se atacan los problemas de las representaciones mentales, de cómo el cuerpo "moldea la mente" (Gallagher 2006) y el del cimiento de los símbolos, o sea de cómo explicar que las representaciones adquieran significados. En el sentido de dar este fundamento a los símbolos, el cuerpo adquiere gran relevancia, pues es a través de los órganos sensoriales y el sistema músculoesquelético que actuamos y nos involucramos con los objetos y el ambiente; si no sentimos y actuamos, el pensamiento está vacío. Aunque esta forma de pensar la cognición ha tomado gran revuelo, hay diversos compromisos respecto a los supuestos y al grado de controversia que se involucra en contraste con las nociones tradicionales. Existen varios asuntos que hay que tomar en cuenta si es que queremos entender y utilizar la cognición corporizada. Si suponemos que podemos dar un modelo en el que la cognición sea sensomotora en su núcleo, no se sigue que la cognición deba ser corporizada en el sentido de requerir un cuerpo para su realización. Pensemos en que cuando se da una cognición en 
línea necesitamos del cuerpo imbuido en el ambiente, sin embargo, cuando lo hacemos fuera de línea (como rememorar, especular, planear) no necesitamos el cuerpo, aunque sí necesitamos la dependencia de las funciones cognitivas del cerebro en la ausencia de inputs sensoriales y outputs motores, de manera que el cuerpo está implicado indirectamente. Lo que esto quiere decir es que las capacidades ostensibles extra motoras como la comprensión léxica dependen hasta cierto punto de funciones cerebrales motoras, lo cual es un pilar de los enfoques corporizados acerca cómo formamos conceptos cómo categorizamos.

Esto implica que no todas las formas de corporización tienen una dependencia corporal en el sentido estricto. Así, en realidad nos enfocamos en la idea de que la cognición depende del cerebro sensomotor, con o sin la presencia directa del cuerpo para la tarea cognitiva. Shaun Gallagher propone una distinción interesante en este sentido, proponiendo los conceptos de imagen y esquema mental:

\begin{abstract}
Una imagen mental es un sistema de percepciones, actitudes y creencias que pertenecen a nuestro cuerpo, una capacidad representacional compleja que es realizada por estructuras en el cerebro. Un esquema mental, por otro lado, incluye capacidades motoras, habilidades y hábitos que al mismo tiempo permiten y constriñen el movimiento y el mantenimiento de la postura, mucho de lo cual no es representacional en carácter ni reductible a funciones mentales (Gallagher 2006, p. 12).
\end{abstract}

Esta distinción permite dar cuenta de que algunos procesos dependen directamente de lo que está ocurriendo en el cuerpo (esquema mental) sin que necesariamente se forme como una representación mental, mientras que otros procesos no requieren de manera directa del cuerpo y operan más con las llamadas imágenes mentales.

\title{
2.1.2. Cognición situada y acción corporizada: dos caras de la moneda
}

Estos dos términos se usan como términos sombrilla que muchas veces se refieren a aproximaciones recientes de todas las disciplinas de las ciencias cognitivas que están relacionadas vagamente. Trataremos a continuación de separarlas un poco. Las aproximaciones corporizadas o encarnadas sostienen que la cognición "depende de los tipos de experiencia que provienen de tener un cuerpo con varias capacidades sensomotrices" (Varela 1991, p. 73). Tomando en cuenta los trabajos que hace el cuerpo en acción también lleva a re-pensar la función de los procesos cognitivos: la cognición se conceptualiza como procesos en tiempo real que coordinan la acción y la percepción en vez de pensarla como el intermedio entre la percepción y la acción para computar soluciones correctas a problemas abstractos.

La unión de la acción corporizada con la cognición situada fue en un inicio la oposición a la mirada tradicional de la ciencia cognitiva, sobre todo al enfatizar el comportamiento observable y las relaciones con el ambiente en vez de los procesos internos de inferencia. Debido a que sus marcos conceptuales se complementan, hay que tener cuidado en esta unidad que se les ha dado, por ejemplo, las aproximaciones encarnadas usualmente se enfocan en las complejidades de los ciclos de acciónpercepción y su inmediata capa ambiental. En contraste, las aproximaciones situadas tienden a enfocarse en relaciones a gran escala de actividades mediadas culturalmente con el pasado, el presente y el futuro de motivaciones personales y desarrollos socio-culturales. En concreto, divergen en su perspectiva de comportamiento (local o global), en su marco conceptual y en su base de estudios de laboratorio y de campo, respectivamente.

Podríamos decir entonces que la cognición situada tiene ciertas características y para ello enumeraremos las que Margaret Wilson (2002, p. 626) propone para ella, es decir, para la cognición que ocurre en el contexto de un ambiente en un mundo real, que inherentemente incluye percepción y acción:

1) La cognición tiene presión temporal: la cognición debe ser entendida bajo las presiones reales del tiempo cuando se interactúa con el ambiente.

2) Le cargamos trabajo cognitivo al ambiente: a causa de limitantes en nuestras habilidades para procesar información (límites en la atención y en la memoria de trabajo) explotamos el ambiente para reducir la carga de trabajo cognitivo. Hacemos que el ambiente sostenga 
o incluso manipule la información para nosotros, y recolectamos dicha información en una toma y daca con base en nuestra necesidad.

3) El ambiente es parte del sistema cognitivo: el flujo de información entre la mente y el mundo es tan densa y continua que, para los científicos que estudian la naturaleza de la actividad cognitiva, la mente sola no es una unidad de análisis significativa.

4) La cognición es para la acción: la función de la mente es guiar la acción, los mecanismos cognitivos como la percepción y la memoria deben ser entendidas en términos de su contribución al comportamiento apropiado en una situación.

5) La cognición fuera de línea está basada en el cuerpo: aunque estemos en un momento dado desacoplados del ambiente, la actividad mental está enraizada en mecanismos que evolucionaron de la interacción con el ambiente, esto es mecanismos de procesamiento sensorial y de control motor.

Mirando estas características podemos decir que puesto de manera muy sencilla la cognición situada es aquella que tiene lugar en el contexto de entradas y salidas relevantes para la tarea a llevarse a cabo en un ambiente específico. Es decir, que mientras un proceso cognitivo toma lugar, la información perceptual continúa fluyendo en el sentido de que afecta el procesamiento, además de que la actividad motora se lleva a cabo de manera que afecta el ambiente de manera relevante. Estos puntos de la cognición situada son muy relevantes para entender las posturas ecológicas, de las que se hablará más adelante. Queda claro entonces que la cognición corporizada se distingue de la situada más a un nivel metodológico que a nivel de análisis, pues es casi imposible pensar una cognición encarnada que no sea situada. Por ejemplo, el concepto central de la cognición corporizada son los bucles de acción percepción, mientras que de la cognición situada es el ambiente ecológico; en este caso el nivel de análisis es la acción corporal para la primera y el contexto socio-cultural para la segunda. Estas distinciones son importantes para ver el nivel de análisis que se debe tener cuando se habla de una u otra capacidad cognitiva, aunque en la realidad están mezcladas dinámicamente.

\subsection{Otras exploraciones}

Exploremos ahora otras ideas de la corporización que tienen cabida pues manejan ciertos matices importantes como la teoría de la mente corpórea de Andy Clark (1999) que postula que la actividad de un organismo en su entorno es parte integrante de sus estados mentales. Esto quiere decir que la interacción cuerpo-mundo constituye la mente, y por tanto, la mente no se encuentra desconectada del cuerpo y sus correspondientes actividades, sino que la mente se encuentra extendida por el entorno. Este proceso de interacción o retroalimentación está mediatizado por trayectorias representacionales, esto es, información descargada por agentes humanos cuando constituyen elementos culturales, y en general, proporcionadas por el mundo. La idea es identificar estas trayectorias que se encuentran tanto en la arquitectura del sistema como en el entorno.

Su explicación contrasta con las anteriores pues no se puede abordar con el tipo de arquitectura funcionalista propia del cognitivismo, aquella que se basa en transformaciones seriales de unos símbolos en otros en términos causales; ni con la que expone el conexionismo, la que postula redes de nodos interconectados, donde la información se distribuye. Así, Clark propone que la cognición es ejecutada por una multiplicidad de máquinas virtuales adaptadas de acuerdo a las necesidades del sistema. Es importante notar que el argumento de Clark para defender su tesis ha sido extraído de la robótica, a saber, la teoría de los agentes autónomos. Entendemos por agente autónomo un sistema encarnado, diseñado para satisfacer objetivos internos o externos mediante sus propias acciones en interacción continua, y a largo plazo con el entorno en el que está situado. Hay que recalcar que para Clark, la mente, al no representar exhaustivamente una situación (como los computacionalistas pensaban), utiliza parte de energía en funciones de acoples con su entorno mediante cierta actividad interna que bien podemos llamar representaciones internas. De esta actividad emerge una pauta de organización del sistema sensorio-motriz con capacidad de acomodarse a los cambios en el entorno.

Existen otras propuestas desde la cognición situada, en las propuestas de cognición encarnada y 
embebida, específicamente autores como Raymound Gibbs (2006) y Shaun Gallagher (2006) han retomado el planteamiento del esquema corporal ${ }^{1}$ para buscar resolver el problema de la cognición. Inicialmente, Gallagher plantea que uno de los grandes problemas de la cognición encarnada y embebida, es cómo entender el papel del cuerpo en ella. Él dirá que hay dos formas en que el cuerpo toma lugar, y que permiten la distinción entre imagen corporal y esquema corporal. Mientras que la primera ayudará a responder la cuestión de cómo aparece el cuerpo en el campo perceptual, el segundo permite resolver cómo el cuerpo forma ese campo (Gallagher 2006, Gibbs 2006). La imagen corporal se entiende como las representaciones conscientes del cuerpo como objeto de sentimientos y emociones (es decir, las autopercepciones). Esta envuelve un nivel de experiencia personal del propio cuerpo. El esquema corporal se entenderá como la manera en que el cuerpo se integra activamente en el ambiente, es lo que permite movernos y ajustarnos al mundo, gracias a la retroalimentación a partir de las terminaciones nerviosas sensoriales y los órganos sensoriales que no requiere de que seamos conscientes de ellas al actuar en el mundo. De esta manera, estos autores se cuestionan, el papel de los procesos representacionales, los cuales parecen no tener ningún papel en la interacción (por lo menos a nivel del esquema) del cuerpo con el entorno; de esta manera las representaciones en cierto tipo de conocimiento con el mundo se vuelven innecesarias.

Otra alternativa es la propuesta enactiva de Alva Nöe (2004), que es menos radical que la propuesta del mismo nombre de Francisco Varela. La teoría de Nöe enfatiza el papel del movimiento como determinante de las maneras en que percibimos el mundo. Para este autor, ser quien percibe es conocer implícitamente que el movimiento afecta la estimulación sensorial. La percepción se convierte en una habilidad corporal, se da por medio de la acción y las percepciones varían de acuerdo a cómo nos movemos e interactuamos en el mundo, no se focalizan a un órgano. La postura de Nöe frente a la percepción permite repensar el papel de las representaciones como aquellas instancias que posibilitan un conocimiento estable del mundo, por lo que no niega la existencia de representaciones a la manera de Varela, ya que para él es claro que de alguna manera tenemos un conocimiento en el que podemos confiar. Sin embargo, aboga por que éstas sean re-evaluadas teniendo en cuenta que la percepción es más flexible de lo que parece, que es siempre movible y que la mente es económica, en el sentido de que no considera necesario tener en la cabeza imágenes rígidas del mundo si la información está al alcance de la mano.

La investigación en neuroetología (Chiek 2008, p. 198) ofrece una mirada en la cual los procesos de los organismos están integrados en sus ambientes. La aportación al debate de la cognición situada es que la investigación en neuroetología muestra que hay una fuerte conexión entre el rol computacional de estructuras que crean los organismos y los aspectos cognitivos de los mismos, de manera que el organismo está naturalmente situado. La idea aquí es que las tareas computacionales que en el pasado se relegaban al sistema nervioso se entienden ahora como que tienen mucho que ver con estructuras no neuronales de los organismos, con estructuras de la forma y el diseño como el cuerpo; a esto se refiere con computación morfológica. ${ }^{2}$ Con esto se trata de decir que no es tan claro donde termina la red neuronal y donde comienzan los órganos sensoriales. Lo que no implica que la computación morfológica y la cognición sean procesos reactivos. Más bien son procesos interactivos: el organismo moldea la influencia que el ambiente tiene sobre él de igual manera que el ambiente moldea el organismo y en parte determina su reacción.

Esta visión dada por la computación morfológica está sentada sobre una perspectiva de las representaciones mentales que no requiere que las representaciones sean dentro de la cabeza en su totalidad, es decir, que no están codificadas. Las perspectivas de representaciones codificadas sostienen que las representaciones son copias, guardadas en los procesos neuronales, de objetos del mundo que

\footnotetext{
El interés por el cuerpo para entender la cognición embebida, toma un camino particular al retomar los planteamientos de autores como Merleau-Ponty. En la Fenomenología de la percepción (Merleau-Ponty 1985), este autor afirma que para entender cómo el ser humano aprehende el mundo se debe tener en cuenta que, perceptualmente hablando, el cuerpo funciona como una unidad orgánica. MerleauPonty afirma que es necesario apelar a un conocimiento intrínseco de nuestro cuerpo, el esquema corporal.

2 Es la noción de que muchas veces las computaciones logísticas sofisticadas se logran por la interacción entre el ambiente y algunas características estructurales del cuerpo de un organismo. Es un término muy usado en inteligencia artificial.
} 
esos procesos transforman, recuperan y relacionan con otros para dirigir las acciones de los organismos. En vez de ello la "representación activa", como le llama Yual Chiek, dice que el acto cognitivo toma ventaja de características del mundo y la usa en lugar de las representaciones (Chiek 2008, p. 194). De acuerdo a esto, las representaciones son compartidas entre las características del ambiente y aquellas que ocurren en la cabeza. Esta perspectiva implica entonces que la cognición no ocurre removida del ambiente sino que ocurre dentro del ambiente, que utiliza características del ambiente para completar los circuitos cognitivos, es decir que la cognición está constituida por esta operación de características del ambiente. Las representaciones ayudan entonces cambiando la naturaleza de la interacción entre la cognición y el ambiente. Así, para Chiek la mente situada permite ser también extendida pues la computación morfológica permite a los organismos tener creencias extendidas.

Resumiendo esta sección, la cognición situada enfatiza que una gran mayoría de la actividad cognitiva sucede en el contexto y activamente interactúa con el ambiente; sin embargo desde el campo de la filosofía aún quedan muchas dudas y especulaciones que sin duda hallarán muchas respuestas trabajando de manera interdisciplinaria con otras ciencias como la Antropología, la Biología y la Neurofisiología, por mencionar sólo algunas.

Como bien dice Frans De Waal:

El campo de la cognición "encarnada" todavía está en pañales, pero tiene profundas implicaciones en nuestra visión de las relaciones humanas. Al meternos de manera involuntaria en la piel de quienes nos rodean, sus movimientos y emociones entran en resonancia con nosotros como si se tratara de nuestro propio cuerpo. Esto es lo que nos permite, a nosotros y a otros primates, recrear lo que hemos visto hacer a otros. La resonancia corporal es un proceso principalmente inconsciente e invisible pero a veces se deja ver (de Waal 2009, p. 88).

\subsection{Cognición extendida y enculturada}

Una vez enmarcados en la cognición situada, queda la pregunta de cómo demarcamos a la cognición (mente/cuerpo) y el ambiente. En general podemos agrupar dos grandes maneras de entender que la cognición esté integrada con el ambiente. La primera es la que se suele denominar mente extendida en la cual está la idea de que el artefacto se integra en el sistema cognitivo a través de un tipo correcto de acoplamiento causal (Clark \& Chalmers 2002) y la extensión está a la par con los procesos cognitivos "en la cabeza". La segunda es la llamada cognición enculturada (EnC) que es la idea de que nuestras habilidades cognitivas son transformadas por prácticas culturales (Menary 2012). De esta manera, lo que somos capaces de hacer es aumentado y transformado por la adquisición de prácticas cognitivas que se desarrollan en un ambiente dado. Este es un ejemplo de la construcción y mantenimiento de un nicho cognitivo, pero uno que va más allá de una manera meramente embebida o en andamios. Las prácticas mismas son parte del ciclo del procesamiento cognitivo y no son meramente auxiliares causales de procesos en la cabeza.

Andy Clark y David Chalmers proponen un externalismo activo, basado en el papel activo que el entorno tiene en la consecución de los procesos cognitivos. Por el otro lado, desde la mirada de la cognición enculturada los artefactos por ellos mismos no extienden nuestras habilidades (en contraste a las tesis fuertes de la mente extendida) sino que las prácticas adquiridas para manipular artefactos y la información guardada en ellas extienden nuestras habilidades cognitivas (aumentándolas y transformándolas). A continuación desarrollaré brevemente ambas propuestas. Es importante señalar, sobre todo para la próxima sección, que toda esta discusión va de la mano con la idea de nicho en donde, por ejemplo, John Odling-Smee, Kevin N. Laland y Marcus W. Felman (2003) consideran que un organismo construye su nicho cuando modifica las relaciones entre el organismo y su ambiente en el presente y en la historia filogenética, ya sea modificando los factores físicos, tanto en su localización espacial como en su temporalidad, o al reubicarse en un nuevo hábitat, exponiéndose a nuevos y diferentes factores, para constituir su nueva biogeografía.

La postura radical es la de la mente extendida de Clark y Chalmers, quienes defienden la tesis de que los procesos cognitivos y mentales van más allá de los límites del cráneo y de la piel: hay elementos 
constitutivos de los procesos mentales y cognitivos más allá de los límites de nuestro propio cuerpo. Una tesis semejante fue defendida por Hilary Putnam (1975), pero Clark y Chalmers distinguen y defienden la independencia entre el tipo de externalismo pasivo defendido por Putnam del externalismo activo que ellos propugnan de acuerdo con el cual "las características externas relevantes son activas, desempeñando un papel crucial aquí y ahora. Como se hayan acopladas con el organismo humano, tienen un impacto directo sobre el organismo y su conducta" (Cark \& Chamlers 2002, p. 67).

En estos casos, el organismo humano se ve unido a una entidad externa en una interacción en dos direcciones, que crea un sistema ensamblado que Clark y Chalmers considerarán un sistema cognitivo por derecho propio. Todos los componentes del sistema juegan un papel causal activo, y gobiernan conjuntamente la conducta, del mismo modo que lo hace la cognición usualmente. Si eliminamos el componente externo del sistema de competencia conductual, es como si elimináramos una parte del cerebro mismo. Los factores externos relevantes son activos, tienen un papel crucial en el aquí y ahora. Dado que existe una complementación con el organismo humano, tienen un impacto directo en el organismo y en su conducta. Al reflexionar sobre este tipo de complementariedad nos vemos abocados a un externalismo activo. Así, al entender la cognición como un proceso extendido, no solamente se toma una decisión terminológica, sino que se establece una diferencia significativa en la metodología de la investigación científica, ya que tiene consecuencias ontológicas fuertes.

Para Clark y Chalmers el cerebro biológico de hecho evolucionó y maduró de maneras que tienen que ver constitutivamente con la presencia fiable de un entorno externo manipulable de manera que los sistemas ensamblados con factores externos son parte del conjunto básico de recursos cognitivos que siempre llevamos con nosotros en el mundo. En este sentido se constituye un sistema integrado entre el cuerpo y el ambiente, constituyendo así un nicho, entendido como lo hace Jacob von Uexküll, "el organismo y su mundo forman una profunda unidad. La estructura del organismo determina el horizonte de las posibles estimulaciones y las posibles reacciones que constituyen el curso de la vida animal; si se cambiara el organismo, tendría también que cambiarse este mundo" (Uexküll 1951, p. 54) a este mundo lo llamo Umwelt, mundo circundante, y un submundo de "operando" o "efectos" al que llamó Wirkungswelt; los efectos pueden cambiar la estructura del ambiente o el estado del organismo.

El mundo circundante y el submundo de los efectos pueden integrarse en el concepto de nicho, aunque éste es uno de los conceptos de difícil definición, es indispensable en ciencias como la ecología, la psicología y en general las ciencias de la conducta para las que hoy es primordial entender las diferentes modalidades de interacción del organismo con el ambiente como también es importante entender las dinámicas de estas interacciones.

Hasta aquí de la mente extendida, para ahora contrapuntearlo con una visión enculturada en donde la radicalidad de la extensión de la mente se ve sustituida por las capacidades que me provee el ambiente, lo cual nos acerca más a la visión de la sección siguiente en la que hablaremos de las afordancias. Esta versión de cómo entendemos la cognición en el ambiente me parece que es más adecuada y con menos requerimientos ontológicos que son difíciles de sostener cuando se analizan los sistemas cognitivos en tareas específicas.

Un sistema es una entidad relativamente estable que está compuesta por un conjunto de partes interactuantes que intercambian energía y alunas veces información y materia con el ambiente. Los sistemas cognitivos están determinados por una serie de relaciones entre componentes. Se pueden identificar un número de potenciales relaciones entre componentes: estructurales, funcionales y de comportamiento. Cuando los componentes de un sistema están relacionados estructuralmente pueden compartir propiedades estructurales o pueden involucrar estructuras híbridas que son ubicuas en naturaleza. Los componentes pueden también tener relaciones funcionales como por ejemplo, que un componente pueda depender en la funcionalidad de otro para que él mismo funcione adecuadamente. Dadas ciertas entradas al sistema los componentes se combinan para producir respuestas o salidas de comportamiento.

La integración cognitiva es una posición de Richard Menary (2007) que toma a los sistemas cognitivos como todos integrados que tienen partes interactuantes pero que dichas partes incluyen 
componentes neurales, del cuerpo y del ambiente. Una manera de verlo es fijarse en la coordinación dinámica de los componentes que interactúan en un sistema. Una parte interesante del trabajo dinámico en la cognición es que algunos componentes que interactúan en los sistemas cognitivos algunas veces están localizados espacialmente fuera del sistema nervioso central del organismo. Las prácticas cognitivas como procesos y como estructuras informacionales pueden ser componentes del sistema aunque no estén dentro de la piel del organismo. Las prácticas cognitivas son estas manipulaciones del cuerpo dotadas culturalmente de estructuras informacionales.

Algunas habilidades requieren facultades para que se lleven a cabo. Algunas habilidades cognitivas pueden ser innatas pues dependen de dichas facultades, pero algunas pueden ser adquiridas. Es muy posible que muchas de las habilidades cognitivas sean una mezcla de ambas, que dependan en las funciones sensoriales y cerebrales y en el aprendizaje y el entrenamiento. Los procesos y habilidades están relacionadas en que una habilidad para hacer algo implica procesos que hacen el trabajo causal requerido para el ejercicio de una habilidad. Por extensión Menary entiende que el ejercicio de la habilidad cognitiva en cuestión incluya algunos procesos cognitivos que no estén limitados en el cerebro y en el cuerpo. Los procesos en cuestión deben estar integrados a rutinas de procesamiento para completar tareas cognitivas. La relación no es de causalidad contingente sino de integración cognitiva: parte del conjunto nuclear de rutinas de procesamiento que llevan directamente a completar una tarea.

Para Menary la cognición es más bien enculturada, es decir que son procesos que se llevan a cabo por estructuras de información manipuladas por el cuerpo. La extensión más importante de nuestros procesos cognitivos es la de los procesos que son parte de una práctica cultural. Las prácticas culturales y representaciones pueden meterse en la piel y transformar el procesamiento y la estructura representacional de los circuitos corticales. Este es el caso más claro de la extensión de una habilidad cognitiva: el dentro es transformado para acoplarse con el afuera. En estos casos las rutinas de procesamiento cruzan del mundo al cerebro: nuestras habilidades cognitivas están enculturadas.

Cuando se explica la propuesta integracionista se recurre frecuentemente a la noción de "tarea cognitiva". Por ejemplo: "Nos implicamos en prácticas cognitivas, manipulando vehículos externos, para completar tareas cognitivas y esta es la marca de lo cognitivo" (Menary 2006, p. 330). Y agrega:

Una definición general de una tarea cognitiva puede, fácilmente, resultar vacía y poco útil. Si definimos

la tarea cognitiva como cualquier tarea para cuya realización es necesaria la cognición, entonces casi cualquier tarea será cognitiva. Creo que es más útil pensar en tareas cognitivas como aquellas que implican el ejercicio de una particular capacidad cognitiva, como recordar una fecha, resolver un problema, aprender a conducir, etc. Estas son tareas dónde el ejercicio de las capacidades cognitivas está directamente ligado a su realización satisfactoria (Menary 2006, p. 343).

Vemos, por tanto, que la noción de tarea cognitiva remite a la de capacidad cognitiva, que tampoco es definida, sino ilustrada mediante ejemplos.

Hay que pensar la extensión de nuestras habilidades cognitivas y el carácter cognitivo en términos integracionistas. Para ello muchos autores como Horst Henddriks-Jansen (1996), han usado las ideas que parten de la cognición ecológica y acuden al término de afordancia, acuñado por James J. Gibson (1979) de manera pionera para hablar de la percepción en la acción sin una abstracción previa. La idea básica es que el ambiente ya tiene "provisto" (que es como se traduce el término afford) las posibilidades de interacción con un actor. Estas posibilidades tienen que ver también con lo que se tiene como marco de referencia, con la combinación de cualidades de este marco. Es una noción que ayuda a plantear una postura tipo la de Menary, dejando de lado posturas radicales como la de la mente extendida.

\section{Las afordancias}

Hemos visto hasta ahora que en las posturas situadas existen debates de cómo comprender las demarcaciones entre lo que llamamos el sistema cognitivo y lo que llamamos el ambiente. El término 
'afordancia' se propone aquí como herramienta epistémica y ontológica, ya que ayuda de una manera sencilla a entender los sistemas cognitivos en su relación con el ambiente y plantea una ontología relacional que deja de lado problemas metafísicos en los que se meten posturas como el externalismo activo, la enacción y la enculturación. Veamos brevemente a continuación la manera histórica de entender la afordancia.

Las afordancias son centrales para el enfoque ecológico de la percepción y la acción. Este punto ha quedado claro por varios autores como Eleanor J. Gibson (1982), James J. Gibson (1986), Harry Heft (2001), Turvey (1992) y otros. James J. Gibson (1986, p. 127) acuña el término directamente del verbo afford en inglés, que quiere decir "tener la capacidad de", "permitirse", "estar al alcance de". De esta manera el ambiente de un animal "le permite" ciertas cosas, para ese animal. Pero, se pregunta Stoffregen, ¿qué clase de cosas son "permitidas"? Su respuesta es que los comportamientos.

Michael T. Turvey (1992) enraíza su definición de afordancia en el concepto de disposiciones, particularmente en el hecho de que las disposiciones ocurren en pares. Además, define las afordancias como propiedades del ambiente, lo que lo lleva a tener que postular otra entidad que no sea propiedad del ambiente, la cual le sirva como la "otra" disposición, complementaria del ambiente, la cual es la efectividad, como propiedad del animal. Thomas A. Stoffregen dice que:

\begin{abstract}
Al basar el enfoque de las afordancias en disposiciones, Turvey arguye que puede dar cuenta de la naturaleza prospectiva de la percepción de las afordancias, esto quiere decir que percibir una afordancia es percibir una posibilidad, algo que podría ser más que algo que es. Las disposiciones implican prospectividad, pues son tendencias o posibilidades, no actualidades. Cuando una disposición se vuelve real, se dice que se actualiza [...]. Otra ventaja de definir las oportunidades para la acción en términos de disposiciones es que este enfoque captura la naturaleza complementaria de las oportunidades para la acción; las oportunidades para la acción se refieren a una adecuación complementaria entre el animal y el ambiente, así como una disposición simple implica una disposición complementaria (Stoffregen 2003, p. 118).
\end{abstract}

Sin embargo, Stoffregen va a criticar ciertas propiedades de las disposiciones que le parece no son aptas para definir 'afordancia'. En particular va a criticar un problema con la tercera característica de las propiedades disposicionales que dice: "Los disposicionales nunca fallan de ser actualizados cuando se unen con circunstancias adecuadas. Una disposición y una circunstancia adecuada es igual a la actualización" (Turvey 1992, p. 178). Sin embargo, hay un problema porque, dice Stoffregen, en cualquier circunstancia hay muchas acciones que son posibles pero la mayoría de ellas no suceden, en términos de Turvey diríamos que muchas de ellas no se actualizan, lo cual contradice el ítem 6.3 que acabamos de citar.

Dice Stoffreggen:

Los eventos animados como subir escaleras o leer son diferentes de los eventos inanimados, como la reflexión de la luz, en que no son obligatorios. Para las cosas vivientes, la conjunción de propiedades particulares del animal con propiedades particulares del ambiente no lleva a la actualización involuntaria de la acción afordada. Las afordancias son lo que podemos hacer, no lo que debemos hacer (Stoffregen 2003, p. 119).

Así, Stroffreggen va entonces a definir 'afordancia' como propiedades emergentes entre el ambiente y el animal, que no dependen en ningún sentido de disposiciones. De la mano de esta noción, retomaremos nosotros la de Anthony Chemero. Chemero (2003) dirá que las afordancias no son propiedades del ambiente, de hecho va más allá y dice que no son propiedades, sino que más bien son relaciones entre aspectos particulares de los animales y aspectos particulares de situaciones.

Para argüir en contra de que las afordancias son propiedades del ambiente lo hace en dos partes. Primero arguye que las afordancias no son siempre propiedades y segundo, arguye que las afordancias no están en el ambiente. La primera es importante en el sentido de que percibir una afordancia es en realidad situar unas características en una situación dada, pero las características no son propiedades de las cosas como tales, sino que surgen de un contexto dado. De aquí es fácil seguir al segundo argumento, ya que si las afordancias son características de situaciones como un todo, los animales son 
en general parte crucial de dicha situación, de manera que percibir algo acerca de la situación no puede ser sólo acerca del ambiente, divorciado del animal (Chemero 2003, p. 185). De esta manera como Stoffregen (2003) sugiere, las afordancias deben pertenecer al sistema animal-ambiente, y no solo al ambiente. Sin embargo, Chemero propone algo más específico que Stoffregen, esto es, que las afordancias son relaciones.

Estas relaciones dicen varias cosas: "percibir afordancias es colocar características, ver que la situación permite una cierta actividad [...]. Las afordancias entonces, son relaciones entre las habilidades del organismo y características del ambiente" (Chemero 2003, pp. 187-189). Es importante aclarar que las habilidades no son disposiciones en el sentido de Turvey (1992). El problema con ver las habilidades como disposiciones es que, cuando están acopladas con las condiciones pertinentes, se garantiza que las disposiciones se vuelvan manifiestas. Pero esto no siempre ocurre ya que, por ejemplo, tener la habilidad de caminar no significa que uno no se caiga aun cuando haya las condiciones ideales para ello. Es mejor entonces entender a las habilidades como propiedades funcionales de los animales (Chemero 2003, pp. 189-190). Las propiedades funcionales de los animales dependen en la historia del desarrollo individual del animal o en la historia evolutiva de la especie. Esto además une a las afordancias con la evolución:

Las afordancias, que son el pegamento que mantiene unidos al animal y al ambiente, existen sólo en virtud de la presión selectiva ejercida en animales por el ambiente físico normal. Surgen junto con las habilidades de los animales de percibir y de tomar ventajas en el ambiente (Chemero 2003, p. 190).

Concretando, las afordancias no son propiedades solas ni del animal ni del ambiente, en vez de eso son relaciones entre las habilidades del animal y algunas características de una situación dada. No son localizables fácilmente físicamente pero son perfectamente reales y percibibles.

Tomando así la noción de afordancia se convierte en una herramienta epistémica que explica la relación del ambiente y el organismo sin tener que tropezarse con problemáticas ontológicas del estilo de la mente extendida que vimos más atrás. Además, esta noción relacional que ofrece Chemero sirve a nuestro punto de vista para explicar fenómenos que se dan precisamente en estas fronteras difusas entre los sistemas cognitivos y el ambiente por su aplicación para fenómenos cognitivos, particularmente en la percepción y la cognición social. En este sentido, percibir afordancias es percibir las propias posibilidades de acción que dependen de las propias capacidades de acción (esto por parte del organismo) en relación a aspectos particulares del ambiente ecológico, el cual incluye aspectos físicos, intencionales e institucionales. Existe además un factor relevante y que se menciona poco en la literatura de las afordancias y es el rol que la cognición social juega en el aprendizaje social, que es comúnmente requerido para entender las posibilidades de acción que se tienen en aspectos particulares del ambiente ecológico (Kaufmann \& Clément 2007). Esto quiere decir que la cognición social moldea en gran sentido la manera en que se da la percepción de un individuo de las afordancias ecológicas, ya que puede determinar si un individuo es capaz o no de usar ciertas afordancias que percibe.

En las últimas décadas, se ha dado una gran cantidad de evidencia que muestra que hay neuronas en la corteza premotora de primates humanos y no humanos que responden al estímulo visual. En particular se ha mostrado que una gran parte de neuronas en el área premotora transforma las propiedades mostradas de los objetos en patrones apropiados para la ejecución motora (Rizzolatti et al. 1988).

Esto revela que la presencia de una conexión directa entre las áreas visuales que reciben información de la retina y las áreas somatosensoriales del circuito parietofrontal. Más precisamente, el camino ventro dorsal explota un sistema de unión directa que automáticamente mapea la información contenida en el estímulo perceptual de un plan específico para la acción (Gallese \& Sinigaglia 2011, p. 516).

Así, como la estimulación perceptual lleva suficiente información para activar somatotópicamente el sistema sensomotor, es posible asumir que este procesamiento subyace la detección directa de patrones sensomotrices en el estímulo. 
Algo que es muy interesante y que describe Silvano Zipoli Caiani es que la evidencia experimental muestra que "solamente mirar una imagen de un objeto resulta en la activación de los planes motores relacionados para la ejecución de acciones basadas en las competencias motoras del observador" (Caiani 2014, p. 283). Lo que esto quiere decir es que la información para la acción puede ser extraída por el sistema visual aun cuando las afordancias de los objetos sean irrelevantes para el sujeto, es decir que de acuerdo a varios experimentos neurobiológicos se apoya la hipótesis de que imágenes de objetos presentadas visualmente activan las mismas áreas sensomotoras que están funcionalmente involucradas en la ejecución de acciones de afordancias de los objetos mostrados. Siguiendo estas evidencias, se puede ver que el sistema perceptual de primates humanos y no humanos es sensible a patrones sensomotores en el estímulo, independientemente de que esté de hecho presente el objeto al que se refiere la acción. Esto quiere decir que el sistema perceptual es sensible a patrones sensomotores que son comunes entre los objetivos reales y los mostrados, digamos en fotos, y no distingue la causa distal de ellas. Esto contradice la idea de que de hecho se requiere la presencia actual de las propiedades disposicionales relacionadas a la acción en el ambiente (como lo planteaba Turvey). En vez de eso lo que se muestra es que lo que se percibe son patrones sensomotrices que portan el estímulo perceptual, en vez de propiedades físicas. En otras palabras, Caiani dice que: "De acuerdo a esto, las afordancias no son propiedades físicas del ambiente pues de hecho no son concebidas como propiedades, sino como relaciones entre patrones sensomotrices transmitidos por los estímulos perceptuales y las habilidades del individuo para actuar" (Caiani 2014, p. 289).

Dependiendo el aspecto percibido por un animal en una situación dada, se pueden estar percibiendo distintos tipos de afordancias, los que tienen que ver con aspectos físicos y que disparan patrones sensomotrices, pero también hay los que serían afordancias sociales o interpersonales que moldean también la manera en la que nos relacionamos con el medio vía otros agentes. Esto es importante pues el rol del aprendizaje social que un ambiente puede ofrecer (en el sentido de Menary diríamos "un ambiente enculturado") se desarrolla en la ontogenia vía la exploración individual y el aprendizaje social en relaciones personales y colectivas.

Nadie nace sabiendo aprovechar lo que el medio "ofrece", es vía estar inmerso en un referente social (enculturados) que podemos tener alcance a actitudes emocionales y atencionales a entidades en el ambiente ecológico. Estas relaciones sociales que se dan desde que somos bebés, van haciendo que nuestro entendimiento del mundo esté dado vía afordancias en términos de relaciones con el ambiente, con otros individuos e incluso con instituciones que se vuelven parte importante para poder interactuar con el ambiente. Todas estas interacciones se pueden entender ontológicamente de una manera relacional que ayuda a deshacerse de problemas como los que plantea la mente extendida, ya que el sistema cognitivo emerge en la consecución de las afordancias, dadas ciertas habilidades (aprendidas socialmente) del individuo con ciertas características del ambiente en situaciones dadas. Queda entonces claro que la cognición social se vuelve un elemento primordial de la posibilidad de relacionarse con el ambiente, de manera que las afordancias ecológicas y sociales (interpersonales) coexisten todo el tiempo.

\section{Conclusiones}

En suma, el término situado puede ser entendido enfatizando los aspectos contextuales, dinámicos, sistemáticos y no localizados de la mente. Puesto concretamente la cognición situada entiende al conocimiento humano no como hechos objetivos sino 1) que surge conceptualmente (construido dinámicamente, recordado, reinterpretado) y articulado en un contexto social; 2) que varía dentro de la población en nichos especializados; 3) socialmente reproducido y 4) transformado por individuos y grupos en procesos de asimilación que son inevitablemente adaptados e interpretados desde perspectivas únicas.

Hemos explicado que el término 'afordancia' se vuelve una excelente herramienta en la explicación de fenómenos de la cognición situada. En particular, epistemológicamente se vuelve una herramienta 
que ayuda a entender los procesos cognitivos como la percepción y la cognición social sin tener que cargar con problemas ontológicos como los de la mente extendida, aunque dando cuenta de fenómenos interesantes al ver a la cognición de esa manera, tales como la idea de entender a las herramientas (como una libreta de apuntes) como parte de un sistema cognitivo. En particular, la afordancia es situada, pero no conlleva un problema ontológico de demarcar al agente del ambiente, sino que más bien logra explicar la relación deshaciéndose de problemas metafísicos que plantean las posturas de la mente extendida y de la cognición enculturada y, sin embargo, tiene un poder explicativo para ambas posturas sin los compromisos ontológicos.

También vimos que en la percepción de afordancias ecológicas debemos tomar el término como uno relacional siguiendo a Chemero (2003). Percibimos el ambiente en términos de la relación entre nuestras habilidades basadas en nuestro cuerpo biológico que es moldeado a través de la interacción social para así, aprender a aprender a sacar provecho del ambiente vía el aprendizaje social. Esto es, percibir afordancias ecológicas sucede en contextos sociales, lo cual quiere decir que sucede en presencia de otras personas, de instituciones, etc. Por consiguiente, la cognición social va moldeando las habilidades de acción percibidas y gran parte de ello se ha mostrado vía experimentos de activación sensomotora. Finalmente, las afordancias sociales y ecológicas se dan en un bucle dinámico en un ambiente dado con ciertas características, recalcando que es una cognición siempre situada y con una ontología relacional, es decir, que los fenómenos cognitivos no se reducen ni al agente ni al ambiente.

Bibliografía

Caiani, S.Z. (2014), "Extending the Notion of Affordance”, Phenomenology and Cognnitive Sciences 13: 275-293.

Clark, A. y D. Chalmers (2002), “The Extended Mind”, en Chalmers, D. (ed.), Philosophy of Mind: Classical and Contemporary Readings, Oxford: Oxford University Press, pp. 643-652.

Clark, A. (1999), Estar ahi. Cerebro, cuerpo y mundo en la nueva ciencia cognitiva, Barcelona: Ediciones Paidós Ibérica S.A..

Chandrasekharan, S. y T.C. Stewart (2007), "The Origin of Epistemic Structures and Proto-Representations”, Adaptive Behaviour 15(3): 329-359.

Chemero, A. (2003), "An Outline of a Theory of Affordances", Ecological Psychology 15(2): 181-195.

Chiek, Y. (2008), "Situated Cognition, the Extended Mind Thesis and the Subject of Cognition", en Hardy-Vallée, B. y N. Payette (eds.), Beyond the Brain: Embodied, Situated and Distributed Cognition, Cambridge: Cambridge Scholars Publishing.

de Waal, F. (2009), La edad de la empatia, México: Tusquets Editores.

Gallagher, S. (2006), How the Body Shapes the Mind, New York: Oxford University Press.

Gallese, V. y C. Sinigaglia (2011), "What is so Special with Embodied Simulation", Trends in Cognitive Science 15(11): 512-519.

Gibson, E.J. (1982), "The Concept of Affordances in Development: The Renascence of Functionalism”, en Collins, W.A. (ed.), The Concept of Development: The Minnesota Symposia on Child Psychology (Vol. 15), New Jersey: Lawrence Erlbaum Associates, pp. 55-81.

Gibson, J.J. ([1979]1986), The Ecological Approach to Visual Perception, New York: Psychology Press, Taylor and Francis Group.

Gibbs, R. (2006), Embodiment and Cognitive Science, Cambridge: Cambridge University Press.

Fodor, J.A. y Z.W. Pylyshyn (2002), "How Direct is Visual Perception?: Some Reflections on Gibson's Ecological Approach”, en Noë, A. y E. Thompson (ed.), Vision and Mind, Cambridge, MA: MIT Press, pp. 167-228.

Heft, H. (2001), Ecological Psychology in Context: James Gibson, Roger Barker, and the legacy of William James's radical empiricism, London: Lawrence Erlbaum Associates. 
Henddriks-Jansen, H. (1996), Catching Ourselves in the Act. Situated Activity, Interactive Emergence, Evolution and Human Thought, Cambridge. MA: MIT Press.

Kaufmann, L y F. Clément (2007), "How Culture Comes to Mind: from Social Affordances to Cultural Analogies", Intellectica 2(46): 221-250.

Menary, R. (2006), “Attacking the Bounds of Cognition”, Philosophical Psychology 19(3): 329-344.

Menary, R. (2007), Cognitive Integration: Mind and Cognition Unbounded, Basingstoke: Palgrave Macmillan.

Menary, R. (2012), “Cognitive Practices and Cognitive Character”, Philosophical Explorations 15(2): 147-164.

Merleau-Ponty, M. (1985), Fenomenología de la percepción, Barcelona: Planeta-Agostini.

Nöe, A. (2004), Action in Perception, Cambridge, MA: The MIT Press.

Odling-Smee, J. J., Laland, K. N. y M. W. Feldman (2003), Niche Construction: The Neglected Process in Evolution, New Jersey: Princeton University Press.

Putnam, H. (1975), “The Meaning of 'Meaning'“, Minnesota Studies in the Philosophy of Science 7: 131-193.

Rizzolatti, G., Camarda, R., Fogassi, L., Gentilucci, M., Luppino, G., y M. Matelli (1988), "Functional Organization of Inferior Area 6 in the Macaque Monkey. II. Area F5 and the Control of Distal Movements”, Experimental Brain Research 71: 491-507.

Stoffregen, T. (2003), "Affordances as Properties of the Animal-Environment System”, Ecological Psychology 15(2): 115134.

Turvey, M.T. (1992), “Affordances and Prospective Control: An Outline of the Ontology”, Ecological Psychology 4: 173. 187.

Uexküll, J. (1951), Ideas para una concepción biológica del mundo, Buenos Aires: Espasa-Calpe.

Varela, T.R. (1991), The Embodied Mind: Cognitive Science and Human Experience, Cambridge, MA: MIT Press.

Wilson, M. (2002), "Six Views of Embodied Cognition”, Psychonomic Bulletin and Review 9(4): 625-236. 